\title{
Phase dependent encapsulation and release profile of ZIF-based biocomposites
}

Received 00th January 20xx, Accepted 00th January 20xx DOI: $10.1039 / x 0 x \times 00000 x$

\author{
F. Carraro, ${ }^{a}$ M. de J. Velasquez-Hernandez, ${ }^{a}$ E. Astria, ${ }^{a}$ W. Liang, ${ }^{b}$ L. Twight, $^{a}$ C. Parise, ${ }^{a, c, d}$ M. Ge, ${ }^{e} Z$. \\ Huang, ${ }^{e}$ R. Ricco, ${ }^{a}$ X. Zou, ${ }^{e}$ L. Villanova ${ }^{f}$ C. Oliver Kappe, ${ }^{c}$ C. Doonan ${ }^{* b}$, P. Falcaro $* a, b$
}

\section{Introduction}

Metal-Organic Frameworks (MOFs) are a class of extended materials synthesized via a modular approach from inorganic (metal clusters or ions) and organic components that typically possess high surface areas and pore volumes. ${ }^{1}$ By carefully selecting the framework building units and reaction conditions the chemistry, porosity and particle size of MOF can be precisely controlled. These properties have attracted researchers to explore MOFs, and their composites, for a wide variety of applications including biomedicine. ${ }^{2}$ For example, MOF particles have shown unprecedented properties for the uptake and release of synthetic drugs, 3,4 and more recently have been integrated with fragile biotherapeutics ${ }^{5,6}$ to improve their stability. ${ }^{7,8}$

a. Institute of Physical and Theoretical Chemistry, Graz University of Technology, Stremayrgasse 9, Graz 8010, Austria

b. Department of Chemistry and the Centre for Advanced Nanomaterials, The University of Adelaide, Adelaide, South Australia 5005, Australia

c. Institute of Chemistry, University of Graz, NAWI Graz, Heinrichstrasse 28, 8010 Graz, Austria

d. Dipartimento di Chimica Industriale "Toso Montanari", Universita' di Bologna, Viale del Risorgimento 4, Bologna

e. Department of Materials and Environmental Chemistry, Stockholm University, 10691 Stockholm, Sweden

f. Faculty of Technical Chemistry, Chemical and Process Engineering, Biotechnology, Graz University of Technology, Petersgasse 10-12, 8010 Graz, Austria

Electronic Supplementary Information (ESI) available: Experimental Section, Tables S1-6, Fig. S1-9, The "ZIF phase analysis" application description. See DOI: $10.1039 / \times 0 \times x 00000 x$
Recently, Zeolitic Imidazolate Frameworks (ZIFs) ${ }^{9,10}$ were used to encapsulate biomacromolecules and to form bio-active composites. ${ }^{11-16}$ The most explored ZIF material for the encapsulation of bioentities is ZIF- 8 which is composed of $\mathrm{Zn}^{2+}$ cations and 2-methylimidazole ( $\mathrm{HmIM})$. ZIF-8-based biocomposites form spontaneously in water around negatively charged biomacromolecules without any additives. ${ }^{17}$ This specific process has been termed biomimetic mineralization due to its broad similarities to naturally occurring biomineralization. ${ }^{11,18,19}$ The ZIF matrix has been shown to protect fragile biomacromolecules and assemblies thereof (e.g. viruses and living cells) from conditions that typically lead to loss of their activity and also act as a vector for in vitro and in vivo delivery. ${ }^{11,12,15,19-23}$ With respect to drug delivery applications, the biomimetic mineralization approach yields high encapsulation efficiencies (EE\%) for biomacromolecules, typically ranging from $80 \%$ to $100 \% .{ }^{11,18,21}$ In general, high EE\% values are relevant to drug delivery applications as the therapeutic is the valuable component of the composite. ${ }^{18}$ Release of the biomolecules is achieved via decomposition of the ZIF- 8 matrix at $\mathrm{pH}$ values $<6.5$, in the presence of chelating agents (e.g. ethylenediaminetetraacetic acid, EDTA), or in specific buffer solutions (e.g. phosphate-buffered saline, PBS). ${ }^{18,24,25}$

ZIF-8 is a crystalline microporous material with sodalite (sod) topology that is synthesized by mixing aqueous solutions of HmIM and $\mathrm{Zn}^{2+} .{ }^{26-28}$ However, by varying the synthetic conditions, other topologies can be obtained (e.g. diamondoid (dia), katsenite (kat), ZIF-L). ${ }^{29-33}$ Similarly, for ZIF-based biocomposites, a variety of topologies can be accessed by 
modulating the reaction conditions. ${ }^{19,34}$ In these studies, a fixed amount of biomacromolecule was employed while the concentrations of the ZIF components were varied. Thus, the network topology was controlled by the relative amount of ligand and cation. In a subsequent study we observed that increasing the biomolecule concentration (i.e. carbohydrates) and maintaining a fixed $\mathrm{HmIM}: \mathrm{Zn}^{2+}$ ratio also led to a change in topology from dia to sod. ${ }^{18}$

This was most likely due to the dependence of sugar concentration on the $\mathrm{pH}$ of the reaction solution as the final solid ZIF product did not contain any carbohydrate. In addition to varying the relative concentration of the ZIF components and biomacromolecules, we have also found that post synthesis treatments (e.g. washing procedure) can trigger phase transitions. ${ }^{18,34}$

Though the various ZIF topologies share the same chemical connectivity, they can exhibit vastly different physical and chemical properties. For example, ZIF (sod) has an accessible porosity of $c a .1800 \mathrm{~m}^{2} \mathrm{~g}^{-1},{ }^{28}$ while ZIF (dia) is non porous to $\mathrm{N}_{2} \cdot{ }^{32}$ In addition, each topology possesses a distinct density and surface chemistry which influences their chemical stability. ${ }^{35,36}$ Accordingly, for biomedical applications, such as drug delivery, we posit that the precise control of topology is critical for the design of a carrier with specific release profiles. In this present work, for the first time, we systematically explored how the combination of the ratio of ZIF components, biomolecule concentration and washing procedure determines the structural phase of the biocomposite.

Here, we screened 36 compositions varying the weight fractions protein for the preparation of biocomposites. ${ }^{37-39}$ The washing procedure was carried out using either water only or water and ethanol. The resulting solids were analysed by X-ray diffraction (XRD) and their topologies represented in ternary phase diagrams namely $\mathrm{TD}-\mathrm{H}_{2} \mathrm{O}$ (water washed) and $\mathrm{TD}-\mathrm{EtOH}$ (water and ethanol washed). A noteworthy result of this study is that we identified proteins encapsulated within $\mathrm{ZIF}-\mathrm{CO}_{3}-1$, a $\mathrm{ZIF}$ previously obtained using solvothermal synthesis in absence of biomacromolecules. ${ }^{40}$ For each distinct phase: amorphous, U13, sodalite, diamondoid, and ZIF- $\mathrm{CO}_{3}-1$ (here referred as am, U13, sod, dia and ZIF-C, respectively) we selected and characterized a representative sample using scanning electron microscopy (SEM), vibrational spectroscopy (Fourier Transformed Infrared, FTIR and Raman), and energy-dispersive X-ray spectroscopy (EDX).

Given the potential application of these biocomposites to drug delivery, for each identified phase we determined the encapsulation and release profiles of BSA and insulin (a clinical bio-therapeutic). Our results show that each of the biocomposites have high encapsulation efficiencies EE\% and distinct release profiles. These data will inform and facilitate future research in the burgeoning area of MOF-based drug delivery.

\section{Results and discussion}

We prepared 36 different samples by varying the composition of zinc acetate, HmIM and BSA (each restricted to a mass

\section{b Ternary Phase Diagram Ethanol (TD-EtOH)}

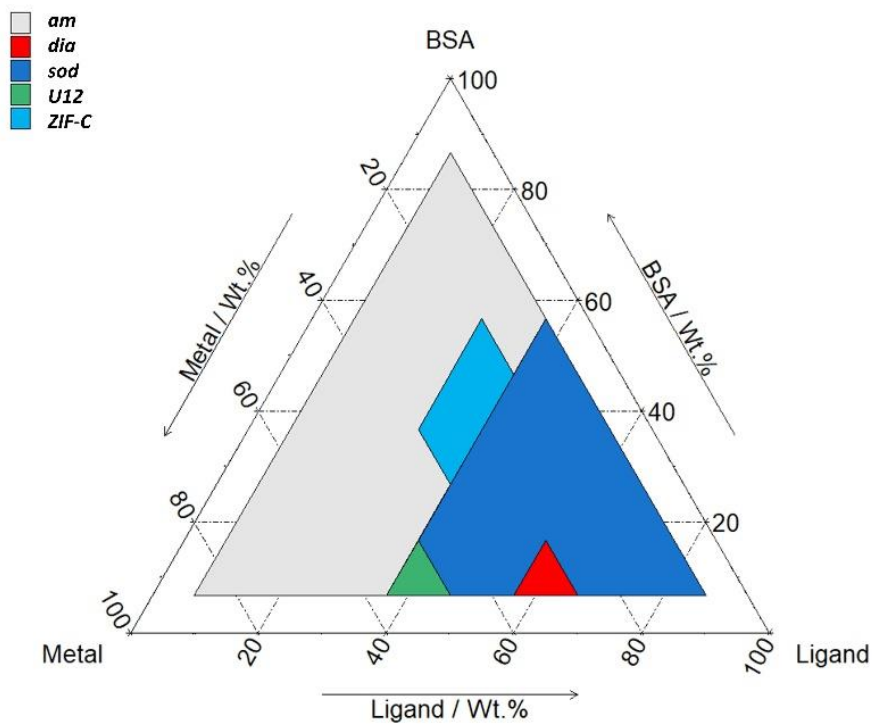

Figure 1: Ternary diagrams (by weight fraction, see Table $\mathrm{S} 1$ for details) of $\mathrm{BSA}, \mathrm{HmIM}$ (labelled as Ligand) and $\mathrm{Zn}\left(\mathrm{OAC}_{2} \cdot 2 \mathrm{H}_{2} \mathrm{O}\right.$ (labelled as Metal). TD- $\mathrm{H}_{2} \mathrm{O}$ (a) represents the main phases (>50\% wt, see Table S2 for details) obtained by washing the sample with DI water. TD-EtOH (b) represents the main phases (>50\% wt, see Table S3 for details) obtained by washing the sample first with DI water and then with ethanol. The total mass of the reagent was chosen selecting a value in between those previous reported in the literature (see ESIt for further details). ${ }^{11,34}$

of $\mathrm{HmIM}, \mathrm{Zn}(\mathrm{OAc})_{2} \cdot 2 \mathrm{H}_{2} \mathrm{O}$, and Bovine Serum Albumin (BSA). BSA was selected as a model biomacromolecule since it has been widely employed in the literature as a standard, inexpensive, 


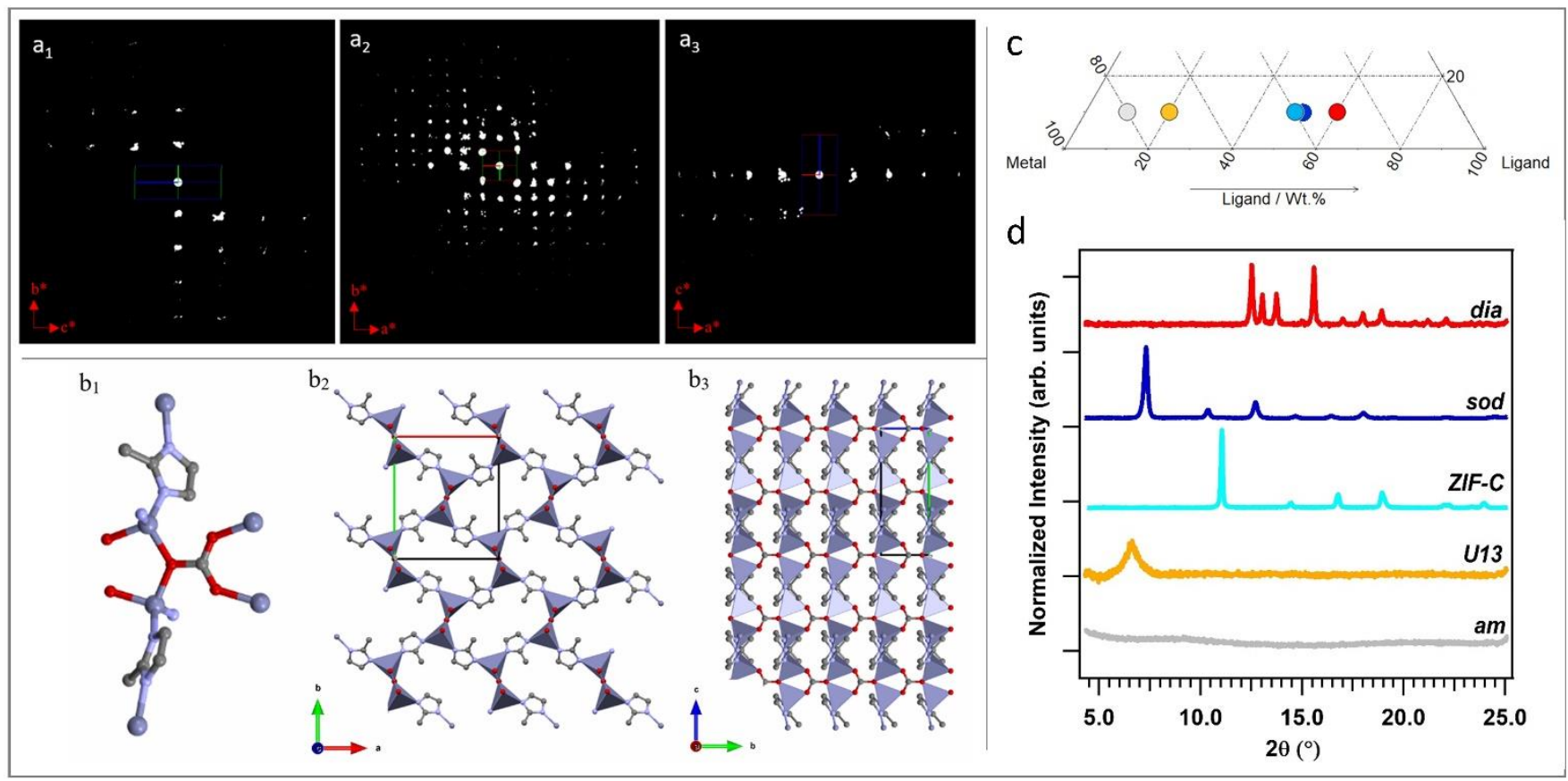

Figure 2: $2 D$ slice cuts from the reconstructed 3D reciprocal lattice show the $0 \mathrm{kl}\left(a_{1}\right)$ hkO $\left(a_{2}\right)$ and hol $\left(a_{3}\right)$ planes. The scattering background (see Fig. S2) was removed for clarification. Structure of ZIF-CO3-1: $\left(b_{1}\right)$ coordination mode of $\mathrm{Zn} ;\left(b_{2}\right)$ framework viewed along the $c$ axis; $\left(b_{3}\right)$ framework structure viewed along the a axis. The $\mathrm{Zn}, \mathrm{O}, \mathrm{N}$ and $\mathrm{C}$ atoms are shown in blue, red, light blue and grey, respectively. c) Section of the ternary diagram to highlight the samples selected as representative of the different phases (grey spot: amorphous biocomposite (TD- $\left.\mathrm{H}_{2} \mathrm{O}\right)$; yellow spot: U13 (TD- $\left.\mathrm{H}_{2} \mathrm{O}\right)$; azure: ZIF-C (TD- $\left.\mathrm{H}_{2} \mathrm{O}\right)$; blue: sod (TD-EtOH); red: dia (TD-EtOH)). The ZIF-C and sod samples were obtained with the same protocol, but with different washing procedures (TD- $\mathrm{H}_{2} \mathrm{O}$ and TD-EtOH, respectively). d) XRD patterns of the amorphous biocomposite and of the biocomposites with dia, sod, ZIF-C topologies, and U13.

fraction range of $10-80 \%$, see Fig. S1 and Tab. S1 in ESIt) in a fixed volume of water ( $2 \mathrm{~mL}$, see ESIt for experimental details) and examined their topology via XRD.

To explore the entire space of the variables, we selected compositions that were equally distributed within the phase diagram plot (see Fig. S1 in ESI + ).

After mixing, the different solutions were left to stand at room temperature for $24 \mathrm{~h}$. From each of the 36 vials, the solid was separated via centrifugation and divided in two parts. One part was washed with deionized (DI) water only and the other was washed with water and ethanol. The samples were then air dried and investigated by X-ray diffraction (XRD). The resultant phases are reported in the ternary diagrams that relate each polymorph to the relative composition of $\mathrm{Zn}(\mathrm{OAc})_{2}, \mathrm{HmIM}$, and BSA in the synthesis solution. Fig. $1 a$ and $b$ show the ternary diagrams of the samples washed with $\mathrm{DI}$ water $\left(\mathrm{TD}-\mathrm{H}_{2} \mathrm{O}\right)$ and with DI water and ethanol (TD-EtOH), respectively. Specific details related to the washing procedures and measurement conditions are reported in ESIt.

When powders were washed only with DI water (TD- $\left.\mathrm{H}_{2} \mathrm{O}\right)$ we observed the formation of an amorphous product (am) for small mass fractions of HmIM (10-20\%). However, increasing HmIM to $20-30 \%$ while keeping $\mathrm{BSA} \leq 20 \%$, we found crystalline patterns dominated by a phase we have previously identified as U13. ${ }^{34}$ Moving towards lower mass fractions of $\mathrm{Zn}^{2+}(10 \%)$, we measured amorphous diffraction patterns until BSA exceeded $50 \%$. The remaining mass fraction ratios yielded diffraction patterns attributed to ZIF-C (Fig. 1a and Fig. S1, Tab. S2 in ESI †). This was confirmed by applying continuous rotation electron diffraction (cRED) technique (Fig. 2a, see ESIt for further details), which is a specialized technique for the structural determination of nanocrystals. ${ }^{41,42}$ ZIF-C is a high density framework (Fig. 2b) non porous to $\mathrm{N}_{2}$ (see Fig. S3, ESI + ), that is prepared using solvothermal conditions $\left(\mathrm{DMF} / \mathrm{H}_{2} \mathrm{O}, 140{ }^{\circ} \mathrm{C}\right)^{40}$ and not observed as a component of a biocomposites until this work. In a limited number of samples, ZIF-C was found mixed with previously reported patterns termed U12, U13, and dia topology. ${ }^{34}$

The TD-EtOH diagram indicates that ethanol washing gives rise to phase transitions: $\boldsymbol{U 1 3}$ is no longer present and all the other samples with ZIF-C are partially or totally converted into sod, with the exception of the dia/ZIF-C mixed phase (TD- $\mathrm{H}_{2} \mathrm{O}$ $\left.\mathrm{BSA} / \mathrm{HmIM} / \mathrm{Zn}^{2+}=10 \% / 60 \% / 30 \%\right)$ that transforms into dia. Furthermore, $\mathbf{U 1 2}$ is converted to a mixture of 3 phases (U12, sod, ZIF-C). Lastly, for all samples with mass fraction $=10 \%$ of $\mathrm{Zn}^{2+}$ and $\mathrm{HmIM} \geq 40 \%$ we measured pure sod. We observed diffraction patterns (i.e. crystalline) only for ca. $w_{\mathrm{HmIM}} \geq 30 \%$, thus TD-EtOH confirms the important role of HmIM for the preparation of a crystalline material.

Combined TD- $\mathrm{H}_{2} \mathrm{O}$ and TD-EtOH show the presence of 5 different phases (am, sod, dia, U13, ZIF-C) in their pure form or as compositions of phases (see Tables S2, S3 ESI+). It is well known that the physical and chemical properties of ZIFs are dependent on their phase $32,43,44$, thus we were motivated to examine the biomolecule encapsulation and release profiles of each biocomposite. However, first, we characterised each material by XRD, FTIR and Raman Spectroscopies, their elemental distribution via EDX and morphologies by SEM. In 

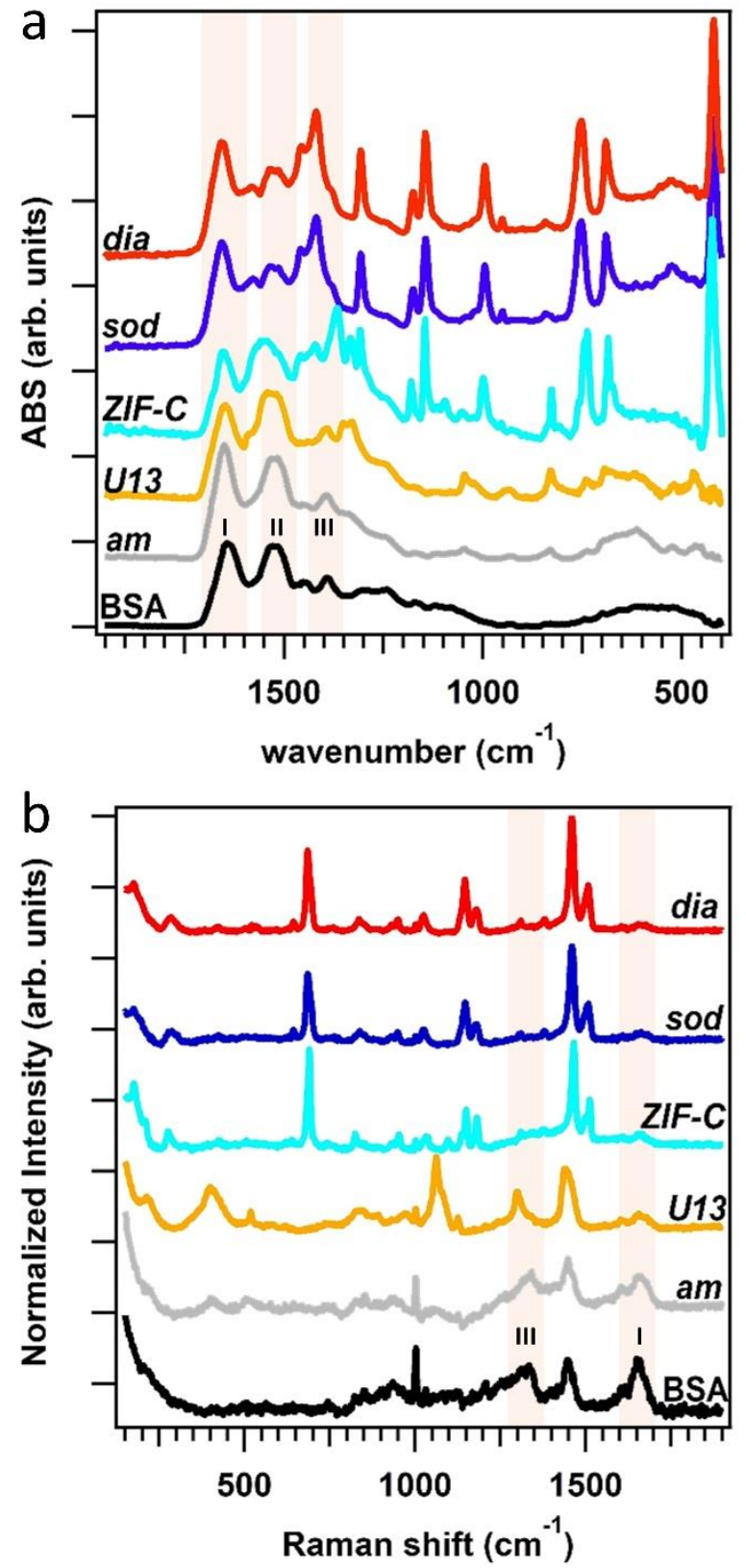

Figure 3: FTIR (a) and Raman (b) spectra of BSA, of the biocomposites with am, dia, sod, U13 and ZIF-C phases. The spectral regions of Amide I, II and III bands of BSA are highlighted in light pink.

addition, given that biopharmaceuticals are the most expensive component of a drug delivery system ${ }^{45-47}$ we selected a biocomposite with a fixed $10 \mathrm{wt} \%$ of protein. Moving along this mass fraction we prepared the 5 different phases shown in Fig. 2c. The diffraction patterns plotted in Fig. $2 d$ are univocally assigned to dia, sod, ZIF-C and U13 (see Fig. S4, ESIt). For am the disordered state is confirmed by the absence of reflections. To facilitate the progress of $\mathrm{Zn}(\mathrm{mIM})_{2}$ bio-composites towards biomedicine and biotechnology, we have developed a web application

https://rapps.tugraz.at/apps/porousbiotech/ZIFphaseanalysis/ ) named ZIF phase analysis. By uploading diffraction patterns collected using $\mathrm{Cu} \mathrm{K} \alpha$ radiation, this web application allows for
1) a rapid identification of the crystalline phases, and 2) a rough estimation of the relative amounts (wt \%). The web application was developed using the statistical software $R$ - shiny package. ${ }^{48}$ Additional information can be found in ESIt.

To assess the connectivity and chemical composition of the biocomposites, we examined powder samples of am, dia, sod, U13 and $\boldsymbol{Z I F - C}$ phases using vibrational spectroscopy (Fig. 3). Analysis of the FTIR data confirms the presence of characteristic modes of the peptide backbone of BSA such as the Amide I (1700-1610 $\left.\mathrm{cm}^{-1}\right)$ and Amide II (1595-1480 $\left.\mathrm{cm}^{-1}\right)$ bands. ${ }^{49,50}$ The spectra of am and U13 did not show vibrational modes that could be attributed to the imidazolate ligand. Furthermore, the vibrational mode at ca. $420 \mathrm{~cm}^{-1}$, assigned to the $\mathrm{Zn}-\mathrm{N}$ stretching mode, is missing in the selected $\boldsymbol{a m}$ and $\boldsymbol{U} \mathbf{1 3}$ samples. This confirms that am and $\boldsymbol{U} 13$ are not $\mathrm{Zn}(\mathrm{mIM})_{2}$-based polymorphs. Conversely, the spectra of sod, dia and ZIF-C show several bands $(420,690,752,998,1145,1175,1308,1419$, $\left.1458,1580 \mathrm{~cm}^{-1}\right)$ typically observed for sod-Zn $(\mathrm{mlM})_{2} \cdot{ }^{31,34,51}$ The spectrum of ZIF-C shows additional bands in the 700-850 and $1300-1400 \mathrm{~cm}^{-1}$ regions that can be assigned to bending and asymmetric stretching modes of $\mathrm{CO}_{3}{ }^{2-} \cdot{ }^{40}$ Moreover, the $\mathrm{Zn}-\mathrm{N}$ stretching mode is slightly shifted from 421 to $427 \mathrm{~cm}^{-1}$; we posit this is due to the different $\mathrm{Zn}$-mIM coordination environment with respect to sod or dia topologies. The Raman spectra $\left(160-1800 \mathrm{~cm}^{-1}\right)$ of the same samples are reported in Fig. 3b. The sod and dia topologies show the typical Raman fingerprint of sod. ${ }^{52,53}$ For both sod and dia, the main bands are assigned to methyl bending $\left(1459 \mathrm{~cm}^{-1}\right), \mathrm{C} 5-\mathrm{N}$ stretching (1147 $\left.\mathrm{cm}^{-1}\right)$, imidazole ring puckering $\left(690 \mathrm{~cm}^{-1}\right)$ and $\mathrm{Zn}-\mathrm{N}$ stretching

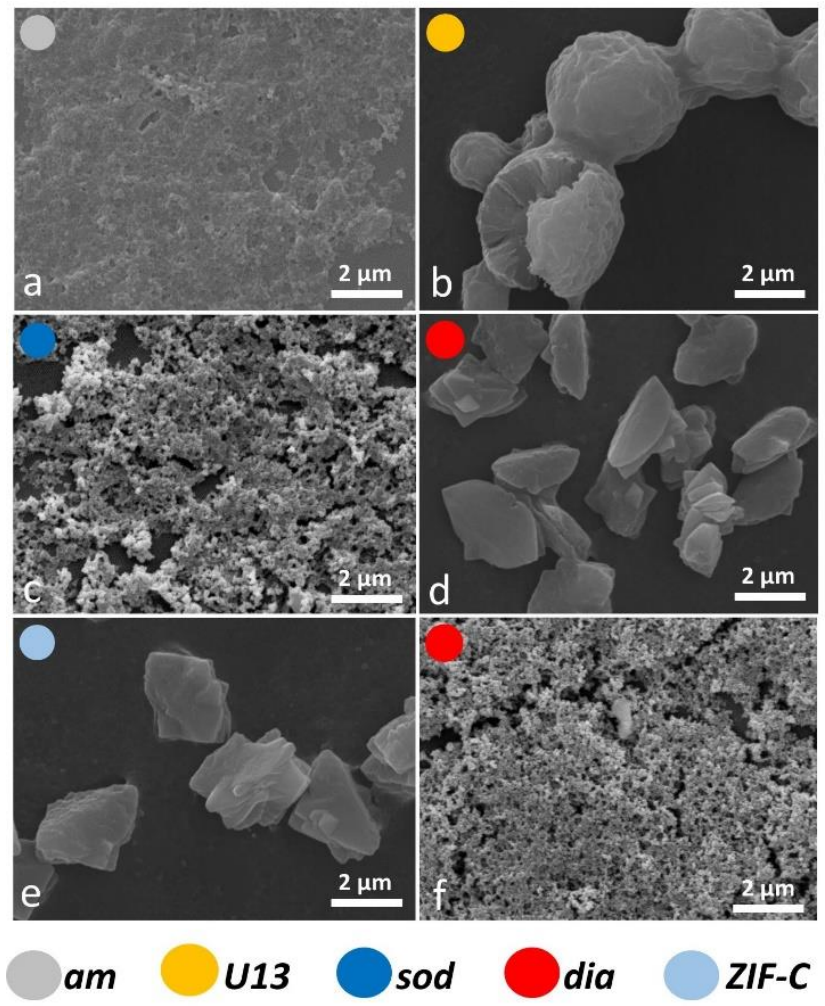

Figure 4: SEM micrographs of the biocomposites with am (a) and U13 ( $b$, from TD$\mathrm{H}_{2} \mathrm{O}$ ), sod (c, from TD-EtOH), dia (from $\mathrm{TD}-\mathrm{H}_{2} \mathrm{O}$ (d) and from TD-EtOH (f)) and ZIF-C (e, from TD- $\mathrm{H}_{2} \mathrm{O}$ ) phases. ZIF-C refers to ZIF-CO $\mathrm{CO}_{3}-1 .^{40}$ 

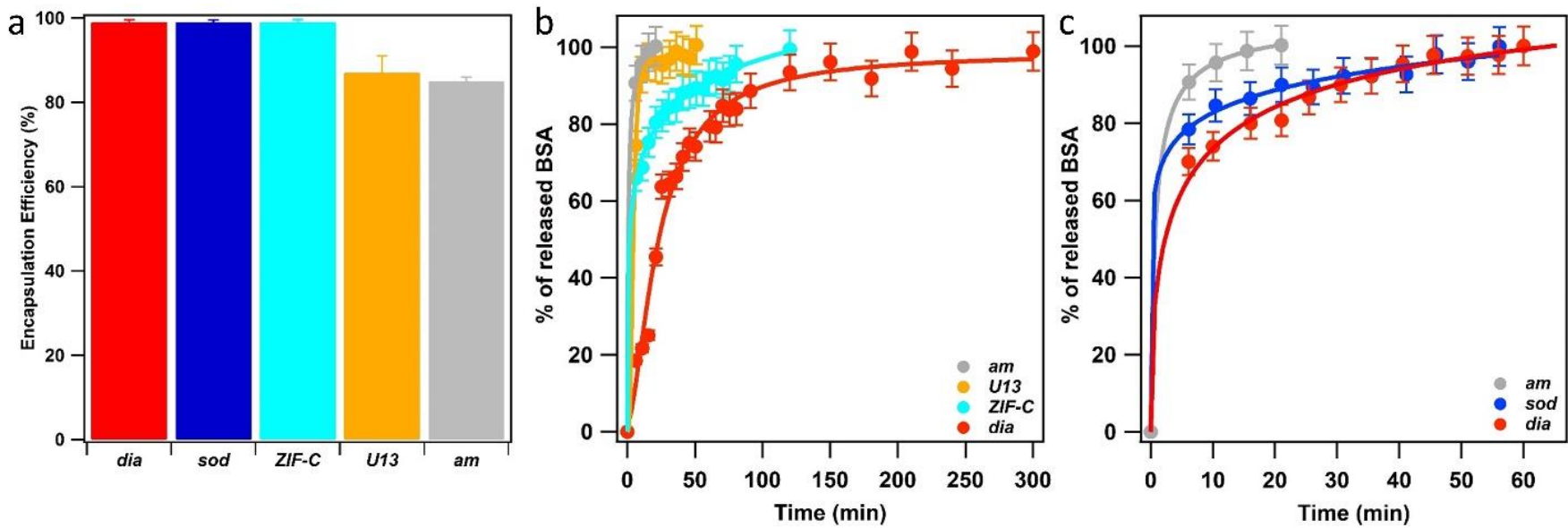

Figure 5: BSA EE\% (a) and BSA release profiles (from TD- $\mathrm{H}_{2} \mathrm{O}$ (b) and from TD-EtOH (c)) of the biocomposites with am, U13, sod, dia and ZIF-C phases. ZIF-C refers to ZIF-CO ${ }_{3}-1.40$

(178 and $\left.278 \mathrm{~cm}^{-1}\right) \cdot{ }^{52,53}$ Comparing the Raman spectra of ZIF-C to sod, small differences can be observed at $1466 \mathrm{~cm}^{-1}$ (assigned to imidazole ring puckering and to methyl bending) and 1097 $\mathrm{cm}^{-1}$ (assigned to $\mathrm{CO}_{3}{ }^{2-}$ stretching mode). ${ }^{52-54}$ These data support the different $\mathrm{Zn}$-mIM coordination environment. In all the three crystalline $\mathrm{Zn}(\mathrm{mIM})_{2}$ phases (sod, dia, ZIF-C) we could confirm the presence of BSA (1550-1720 $\mathrm{cm}^{-1}$, Amide I). ${ }^{55}$ For amorphous and $\boldsymbol{U} \mathbf{1 3}$ the vibrational modes of BSA dominate the spectra with broad bands assigned to Amide I (1600-1700 $\left.\mathrm{cm}^{-1}\right)$, Amide III (1300-1350 cm-1) and -CH deformation $\left(1445 \mathrm{~cm}^{-1}\right) \cdot{ }^{55}$ The broad band at $400 \mathrm{~cm}^{-1}$ could be attributed to $\mathrm{Zn}-\mathrm{O}$ stretching. This indicates potential for zinc protein interactions. $^{56}$ The elemental composition of the biocomposites estimated by EDX shows a Zn content of ca. 5 $w t \%$ in the case of sod. This value increases to ca. $15 \%$ for am and ca. $18 \%$ for $\boldsymbol{U 1 3}$ (details can be found in ESIt, Fig. S5 and Table S5), suggesting that $\boldsymbol{a m}$ and $\boldsymbol{U 1 3}$ are mainly composed of $\mathrm{Zn}$ and BSA. We note that $\mathrm{Zn}$ cations and BSA have been shown to form solid particles. ${ }^{57,58} \mathrm{Next}$, we investigated the morphology of the different phases by SEM (Fig. 4). For the water washed samples, very small particles of indistinguishable morphology were observed for am (Fig. 4a). While for U13 the image shows spherical particles, and for dia- $\mathrm{Zn}(\mathrm{mIM})_{2}$ and $\mathbf{Z I F - C}$ aggregates of plates 2-3 $\mu \mathrm{m}$ in size (Fig. $4 \mathrm{~b}$ and $4 \mathrm{~d}$ and e, respectively). For the ethanol washed sample, the particle size was reduced to less than $100 \mathrm{~nm}$ (Fig. 4c, f and S6, ESIT), with the exception of $U_{13}$. In this case, a similar particle morphology is observed despite the phase transition to am (Fig. S7, ESI+).

We then turned our efforts to investigate the potential of using the 5 biocomposites am, U13, sod, dia, and ZIF-C as drug delivery systems. Initially BSA was employed as a model biotherapeutic. ${ }^{59,60}$ For each sample a $10 \%$ mass fraction of BSA was employed in the synthesis (vide supra) and two important properties of a drug carrier were assessed: $\mathrm{EE} \%$ and release profile. ${ }^{61-63}$ The estimated EE\% (average of five independent analyses) for the different phases is shown in Fig. 5a. For each phase, high EE\% values were observed (EE\%>85\%) and, remarkably, dia, sod and ZIF-C topology showed a 100\% EE. Details can be found in ESI†.

BSA release profiles were investigated to ascertain the quantity of protein released over time. The dissolution of each biocomposite was performed by exposing $1.08 \mathrm{mg}$ of each phase to citric acid buffer solution ( $1 \mathrm{~mL}, 100 \mathrm{mM}$, pH 5.5, room
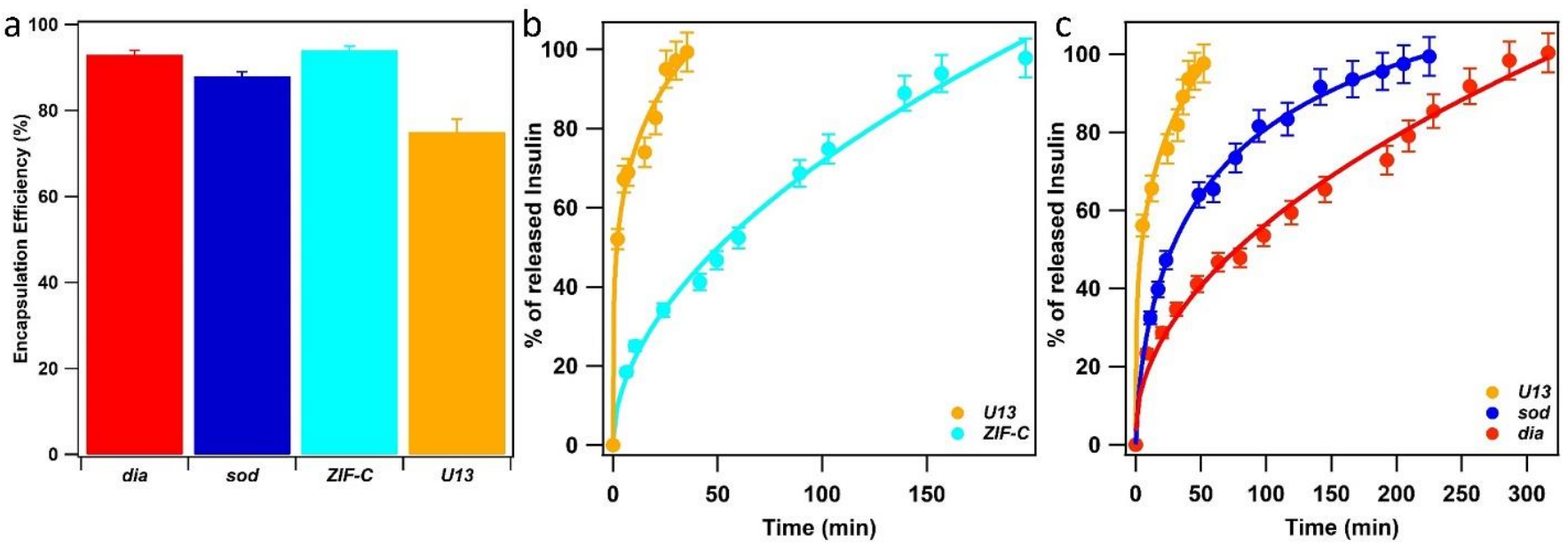

Figure 6: Insulin EE\% (a) and Insulin release profiles of the biocomposites with U13 and ZIF-C phases (b, water washed samples) and U13, sod and dia (c, EtOH washed samples) phases. ZIF-C refers to $\mathrm{ZIF}-\mathrm{CO}_{3}-1.40$ 
temperature). We used UV-Vis and the Bradford Assay (see ESI for further details) to measure the amount of BSA in solution over time (see ESIt for details), and the release profiles are plotted in Fig. $5 \mathrm{~b}$ and c. The experimental data points were fitted with a logistic fitting function ${ }^{64}$ which has previously been employed in the literature for the analysis of data related to the dissolution of different hydrophobic carries, including ZIF8. ${ }^{18,65,66}$ Among the biocomposites washed only with water, am and $\boldsymbol{U} 13$ showed the fastest dissolution: in 20 minutes, $100 \%$ of the encapsulated BSA was released (see ESIT) and the slowest release was measured for the dia topology: $100 \%$ of protein release was reached after 250 minutes. Whilst $\mathbf{Z I F - C}$ released $100 \%$ of the protein in $120 \mathrm{~min}$. With respect to the ethanolwashed samples, am showed the rapid release ca. $20 \mathrm{~min}$ and similar to the water washed samples the slowest release was measured for the biocomposite of dia topology. However, in this case the release was significantly faster; $100 \%$ was observed in 60 minutes (compared to $250 \mathrm{~min}$ for the water washed samples). Given that the topologies are identical for the water and ethanol washed samples we posit that difference in release times is due to the significantly smaller particle size of the ethanol-washed dia biocomposites (100 nm) compared to the water-washed particles $(2 \mu \mathrm{m})$. Thus, we can conclude that both the crystalline phase and the particle size play a crucial role in the design of MOF carriers for drug delivery applications.

To explore the potential biomedical applications of these biocomposites for the delivery of therapeutics we determined the release profiles of insulin encapsulated within the same 5 phases as studied for BSA. XRD data confirmed the expected phases (see Fig. S8, ESIt). However, for the dia phase an impurity of sod was present (15 wt\%). For the samples with dia, sod and ZIF-C topology we measured 93, 88 and $94 \%$ of insulin EE\%, respectively (Fig. 6a). The lowest value was found for $\boldsymbol{U 1 3}$ sample $(E E \%=75 \%)$. Then, we tested the insulin release profiles (Fig.6b and c). Among the crystalline water-washed samples, the $\boldsymbol{U 1 3}$ biocomposite showed the fastest release: in the first 40 minutes $100 \%$ of the encapsulated insulin was released. The slowest release was measured for the sample that possesses ZIF-C topology: the insulin release is $50 \%$ in $60 \mathrm{~min}$ and $100 \%$ in $200 \mathrm{~min}$. Among the ethanol-washed crystalline samples, U13 showed the fastest release and a profile comparable to the water-washed $\mathbf{U 1 3}$ sample. The slowest release was measured for the sample that possesses dia topology: $100 \%$ of release was reached in 300 minutes. The sample that possesses sod topology showed an intermediate release profile $(100 \%$ of insulin released in 220 minutes).

\section{Conclusion}

We investigated the dependence of the crystal phases on the mass fraction of precursors (BSA or insulin, $\mathrm{Zn}(\mathrm{OAC})_{2} \cdot 2 \mathrm{H}_{2} \mathrm{O}$, $\mathrm{HmIM}$ ) and the washing procedure (water or ethanol). For BSA we prepared 36 samples, washed only with water; the crystal phases were used to plot a ternary phase diagram (TD- $\left.\mathrm{H}_{2} \mathrm{O}\right)$. More than $40 \%$ of the samples were found to be amorphous and the remaining samples where crystalline and non-porous (dia, U13, ZIF-C). Then, we tested the effect of ethanol washes on the 36 samples and found that it gave rise to phase transitions. For example, $\mathbf{U 1 3}$ became amorphous, while ZIF-C transformed partially or completely into sod. From these data we constructed a new second ternary phase diagram (TD-EtOH). Approximately $50 \%$ of the samples in TD-EtOH are amorphous; the remaining crystalline samples are dominated by the porous sod topology. The two ternary diagrams were used for the design of BSA-based composites with different crystallinity: starting with the same amount of protein, we could select conditions for the preparation of 5 different crystalline phases. To assess the potential of these systems for application to drug delivery, we focused our attention on determining their encapsulation efficiency and release profiles. We measured encapsulation efficiencies over $85 \%$ and a $100 \%$ release that can be tuned from 20 to 300 min depending on the selected phase. In general, we believe that the here reported ternary diagrams can be used to design new biocomposites with tailored functional properties for bio-catalysis, bio-banking and drug delivery. As a proof of concept, we applied the ternary diagram to synthesize insulin bio-composites and test their encapsulation and release properties. Finally, we uncovered for the first time proteins@ZIF-C composites. For BSA@ZIF-C and insulin@ZIF-C, we measured EE\% ${ }_{\mathrm{BSA}}=99 \%$ and EE\% insulin $=94 \%$, and $100 \%$ release was achieved in 120 and $200 \mathrm{~min}$, respectively. This new bioMOF composite is an appealing crystalline structure alternative to sod and dia with potentially useful properties for encapsulation and release of biotherapeutics.

\section{Conflicts of interest}

There are no conflicts to declare.

\section{Acknowledgements}

The research leading to these results has received funding from the European Research Council under the European Union's Horizon 2020 Programme (FP/2014-2020)/ERC Grant Agreement no. 771834 - POPCRYSTAL. The authors acknowledge support from the European Union's Horizon 2020 FETOPEN-1-2016-2017 research and innovation program under grant agreement 801464 . This work was supported by the Australian Research Council Discovery Project (DP170103531). P. F. acknowledges TU Graz for the Lead Project (LP-03). M. J. V. $\mathrm{H}$. acknowledges The National Council of Science and Technology (CONACYT, México) for the postdoctoral scholarship (CVU 419210). R. R. acknowledges the European Union's Horizon 2020 research and innovation programme under the Marie Skłodowska-Curie grant agreement \#748649 (project "MNEMONIC"). Financial support from the Swedish Research Council (2017-0432) and the Knut and Alice Wallenberg Foundation (KAW 2016.0072) are gratefully acknowledged. 


\section{Notes and references}

1 H. Furukawa, K. E. Cordova, M. O'Keeffe and O. M. Yaghi, Science, 2013, 341, 1230444.

2 A. C. McKinlay, R. E. Morris, P. Horcajada, G. Férey, R. Gref, P. Couvreur and C. Serre, Angewandte Chemie International Edition, 2010, 49, 6260-6266.

3 P. Horcajada, C. Serre, M. Vallet-Regí, M. Sebban, F. Taulelle and G. Férey, Angewandte Chemie International Edition, 2006, 45, 5974-5978.

4 S. Wuttke, A. Zimpel, T. Bein, S. Braig, K. Stoiber, A. Vollmar, D. Müller, K. Haastert-Talini, J. Schaeske, M. Stiesch, G. Zahn, A. Mohmeyer, P. Behrens, O. Eickelberg, D. A. Bölükbas and S. Meiners, Advanced Healthcare Materials, 2017, 6, 1600818.

5 C. Doonan, R. Riccò, K. Liang, D. Bradshaw and P. Falcaro, Accounts of Chemical Research, 2017, 50, 1423-1432.

6 D. A. LaVan, D. M. Lynn and R. Langer, Nat Rev Drug Discov, 2002, 1, 77-84.

7 Y. Chen, P. Li, J. A. Modica, R. J. Drout and O. K. Farha, J. Am. Chem. Soc., 2018, 140, 5678-5681.

8 Y. Feng, H. Wang, S. Zhang, Y. Zhao, J. Gao, Y. Zheng, P. Zhao, Z. Zhang, M. J. Zaworotko, P. Cheng, S. Ma and Y. Chen, Advanced Materials, 2019, 31, 1805148.

9 J. Yao and H. Wang, Chem. Soc. Rev., 2014, 43, 4470-4493.

10 B. Chen, Z. Yang, Y. Zhu and Y. Xia, J. Mater. Chem. A, 2014, 2, 16811-16831.

11 K. Liang, R. Ricco, C. M. Doherty, M. J. Styles, S. Bell, N. Kirby, S. Mudie, D. Haylock, A. J. Hill, C. J. Doonan and P. Falcaro, Nature Communications, , DOI:10.1038/ncomms8240.

12 W. Liang, H. Xu, F. Carraro, N. K. Maddigan, Q. Li, S. G. Bell, D. M. Huang, A. Tarzia, M. B. Solomon, H. Amenitsch, L. Vaccari, C. J. Sumby, P. Falcaro and C. J. Doonan, Journal of the American Chemical Society, 2019, 141, 2348-2355.

13 F. Lyu, Y. Zhang, R. N. Zare, J. Ge and Z. Liu, Nano Lett., 2014, 14 5761-5765.

14 X. Wu, M. Hou and J. Ge, Catalysis Science \& Technology, 2015, 5, 5077-5085.

15 X. Wu, J. Ge, C. Yang, M. Hou and Z. Liu, Chemical Communications, 2015, 51, 13408-13411.

16 F.-K. Shieh, S.-C. Wang, C.-I. Yen, C.-C. Wu, S. Dutta, L.-Y. Chou, J. V. Morabito, P. Hu, M.-H. Hsu, K. C.-W. Wu and C.-K. Tsung, J. Am. Chem. Soc., 2015, 137, 4276-4279.

17 N. K. Maddigan, A. Tarzia, D. M. Huang, C. J. Sumby, S. G. Bell, P. Falcaro and Christian. J. Doonan, Chemical Science, 2018, 9, 4217-4223.

18 E. Astria, M. Thonhofer, R. Ricco, W. Liang, A. Chemelli, A. Tarzia, K. Alt, C. E. Hagemeyer, J. Rattenberger, H. Schroettner, T. Wrodnigg, H. Amenitsch, D. M. Huang, C. J. Doonan and P. Falcaro, Materials Horizons, 2019, 10.1039.C8MH01611A.

19 S. Li, M. Dharmarwardana, R. P. Welch, C. E. Benjamin, A. M. Shamir, S. O. Nielsen and J. J. Gassensmith, ACS Appl. Mater. Interfaces, 2018, 10, 18161-18169.

20 M. Hoop, C. F. Walde, R. Riccò, F. Mushtaq, A. Terzopoulou, X.-Z. Chen, A. J. deMello, C. J. Doonan, P. Falcaro, B. J. Nelson, J. Puigmartí-Luis and S. Pané, Applied Materials Today, 2018, 11, 13-21.

21 T.-T. Chen, J.-T. Yi, Y.-Y. Zhao and X. Chu, Journal of the American Chemical Society, 2018, 140, 9912-9920.

22 W. Chen and C. Wu, Dalton Trans., 2018, 47, 2114-2133.

23 X. Wu, C. Yang and J. Ge, Bioresources and Bioprocessing, 2017, 4, 24.
24 M. de J. Velásquez-Hernández, R. Ricco, F. Carraro, F. T. Limpoco, M. Linares-Moreau, E. Leitner, H. Wiltsche, J. Rattenberger, H. Schröttner, P. Frühwirt, E. M. Stadler, G. Gescheidt, H. Amenitsch, C. J. Doonan and P. Falcaro, CrystEngComm, 2019, 21, 4538-4544.

25 M. A. Luzuriaga, C. E. Benjamin, M. W. Gaertner, H. Lee, F. C. Herbert, S. Mallick and J. J. Gassensmith, Supramolecular Chemistry, 2019, 0, 1-6.

26 O. Karagiaridi, M. B. Lalonde, W. Bury, A. A. Sarjeant, O. K. Farha and J. T. Hupp, J. Am. Chem. Soc., 2012, 134, 18790-18796.

27 Q. Bao, Y. Lou, T. Xing and J. Chen, Inorganic Chemistry Communications, 2013, 37, 170-173.

28 K. Kida, M. Okita, K. Fujita, S. Tanaka and Y. Miyake, CrystEngComm, 2013, 15, 1794.

29 M. Jian, B. Liu, R. Liu, J. Qu, H. Wang and X. Zhang, RSC Advances, 2015, 5, 48433-48441.

30 A. D. Katsenis, A. Puškarić, V. Štrukil, C. Mottillo, P. A. Julien, K. Užarević, M.-H. Pham, T.-O. Do, S. A. J. Kimber, P. Lazić, O. Magdysyuk, R. E. Dinnebier, I. Halasz and T. Friščić, Nature Communications, 2015, 6, 6662.

31 M. He, J. Yao, Q. Liu, K. Wang, F. Chen and H. Wang, Microporous and Mesoporous Materials, 2014, 184, 55-60.

32 Z. Akimbekov, A. D. Katsenis, G. P. Nagabhushana, G. Ayoub, M. Arhangelskis, A. J. Morris, T. Friščić and A. Navrotsky, J. Am. Chem. Soc., 2017, 139, 7952-7957.

33 R. Chen, J. Yao, Q. Gu, S. Smeets, C. Baerlocher, H. Gu, D. Zhu, W. Morris, O. M. Yaghi and H. Wang, Chemical Communications, 2013, 49, 9500-9502.

34 W. Liang, R. Ricco, N. K. Maddigan, R. P. Dickinson, H. Xu, Q. Li, C. J. Sumby, S. G. Bell, P. Falcaro and C. J. Doonan, Chemistry of Materials, 2018, 30, 1069-1077.

35 S. Cao, T. D. Bennett, D. A. Keen, A. L. Goodwin and A. K. Cheetham, Chem. Commun., 2012, 48, 7805-7807.

36 C. Avci, J. Ariñez-Soriano, A. Carné-Sánchez, V. Guillerm, C. Carbonell, I. Imaz and D. Maspoch, Angewandte Chemie International Edition, 2015, 54, 14417-14421.

37 D. J. Connell, A. Gebril, M. A. H. Khan, S. V. Patwardhan, K. Kubiak-Ossowska, V. A. Ferro and P. A. Mulheran, Scientific Reports, 2018, 8, 17115.

38 K. A. Majorek, P. J. Porebski, A. Dayal, M. D. Zimmerman, K. Jablonska, A. J. Stewart, M. Chruszcz and W. Minor, Mol Immunol, 2012, 52, 174-182.

39 W. Liang, F. Carraro, M. B. Solomon, S. G. Bell, H. Amenitsch, C. J. Sumby, N. G. White, P. Falcaro and C. J. Doonan, J. Am. Chem. Soc., 2019, 141, 14298-14305.

40 S. A. Basnayake, J. Su, X. Zou and K. J. Balkus, Inorg. Chem., 2015, 54, 1816-1821.

41 M. O. Cichocka, J. Ångström, B. Wang, X. Zou and S. Smeets, J Appl Crystallogr, 2018, 51, 1652-1661.

42 S. Yuan, J.-S. Qin, H.-Q. Xu, J. Su, D. Rossi, Y. Chen, L. Zhang, C. Lollar, Q. Wang, H.-L. Jiang, D. H. Son, H. Xu, Z. Huang, X. Zou and H.-C. Zhou, ACS Cent. Sci., 2018, 4, 105-111.

43 Y. Lo, C. H. Lam, C.-W. Chang, A.-C. Yang and D.-Y. Kang, RSC Advances, 2016, 6, 89148-89156.

44 L. Bouëssel du Bourg, A. U. Ortiz, A. Boutin and F.-X. Coudert, APL Materials, 2014, 2, 124110.

45 M. Baker, Nat Biotechnol, 2005, 23, 1065-1072.

46 T. W. Overton, Drug Discovery Today, 2014, 19, 590-601.

47 G. Walsh, Nat Biotechnol, 2010, 28, 917-924.

48 W. Chang, J. Cheng, J. J. Allaire, Y. Xie, J. McPherson, RStudio, jQuery F. (jQuery library and jQuery U. library), jQuery contributors (jQuery library; authors listed in 
inst/www/shared/jquery-AUTHORS.txt), jQuery U. contributors (jQuery U. library; authors listed in inst/www/shared/jqueryui/AUTHORS.txt), M. O. (Bootstrap library), J. T. (Bootstrap library), B. contributors (Bootstrap library), Twitter, I. (Bootstrap library), A. F. (html5shiv library), S. J. (Respond js library), S. P. (Bootstrap-datepicker library), A. R. (Bootstrap-datepicker library), D. G. (Font-A. font), B. R. (selectize js library), K. M. K. (es5-shim library), es5-shim contributors (es5-shim library), D. I. (ion rangeSlider library), S. S. (Javascript strftime library), S. L. (DataTables library), J. F. (showdown js library), J. G. (showdown js library), I. S. (highlight js library) and R. C. T. (tar implementation from R), shiny: Web Application Framework for R, 2019.

49 A. Barth, Biochimica et Biophysica Acta (BBA) - Bioenergetics, 2007, 1767, 1073-1101.

50 M. Jackson and H. H. Mantsch, Critical Reviews in Biochemistry and Molecular Biology, 1995, 30, 95-120.

51 Y. Hu, H. Kazemian, S. Rohani, Y. Huang and Y. Song, Chem. Commun., 2011, 47, 12694-12696.

52 G. Kumari, K. Jayaramulu, T. K. Maji and C. Narayana, J. Phys. Chem. A, 2013, 117, 11006-11012.

53 J. Ethiraj, F. Bonino, C. Lamberti and S. Bordiga, Microporous and Mesoporous Materials, 2015, 207, 90-94.

54 G. Socrates, Infrared and Raman Characteristic Group Frequencies: Tables and Charts, John Wiley \& Sons, 2004.

55 N. Kuhar, S. Sil, T. Verma and S. Umapathy, RSC Adv., 2018, 8, 25888-25908.

56 M. Wang, L. Jiang, E. J. Kim and S. H. Hahn, RSC Adv., 2015, 5, 87496-87503.

57 C. Li, L. Xing and S. Che, Dalton Trans., 2012, 41, 3714-3719.

58 H. Qing, H. Yanlin, S. Fenlin and T. Zuyi, Spectrochimica Acta Part A: Molecular and Biomolecular Spectroscopy, 1996, 52, 17951800.

59 A. S. Determan, B. G. Trewyn, V. S.-Y. Lin, M. Nilsen-Hamilton and B. Narasimhan, Journal of Controlled Release, 2004, 100, 97-109.

60 K. N. Clayton, D. Lee, S. T. Wereley and T. L. Kinzer-Ursem, Lab Chip, 2017, 17, 4148-4159.

61 M. Mishra, Handbook of Encapsulation and Controlled Release, CRC Press, 2015.

62 M. L. Hans and A. M. Lowman, Current Opinion in Solid State and Materials Science, 2002, 6, 319-327.

63 C.-Y. Sun, C. Qin, X.-L. Wang and Z.-M. Su, Expert Opinion on Drug Delivery, 2013, 10, 89-101.

64 F. O. Costa, J. J. S. Sousa, A. A. C. C. Pais and S. J. Formosinho, Journal of Controlled Release, 2003, 89, 199-212.

65 K. Zhang, R. P. Lively, C. Zhang, R. R. Chance, W. J. Koros, D. S. Sholl and S. Nair, J. Phys. Chem. Lett., 2013, 4, 3618-3622.

66 K. Ghosal, A. Chandra, R. Rajabalaya, S. Chakraborty and A. Nanda, Pharmazie, 2012, 67(2), 147-55. 
TOC

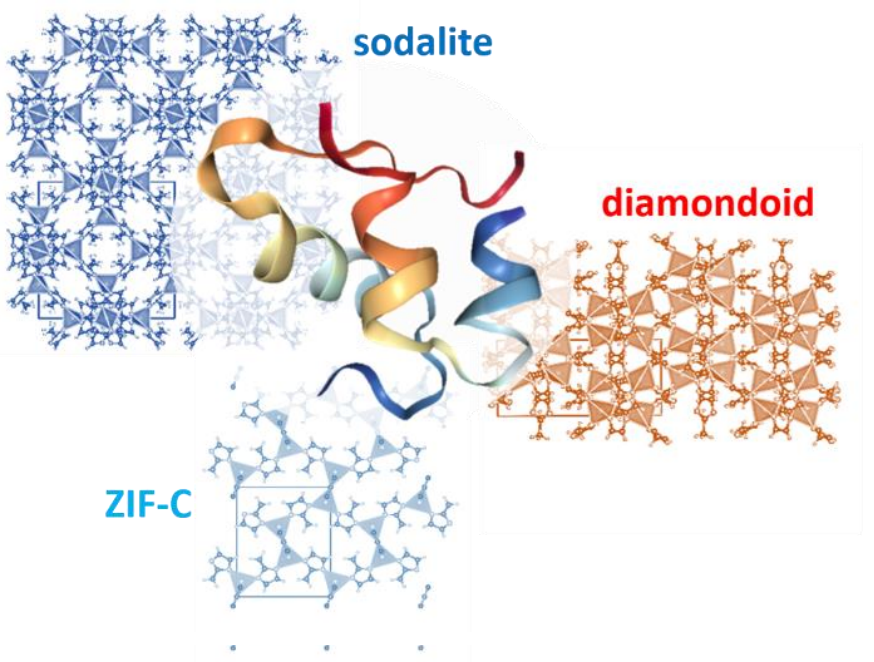

